\title{
Administración de fármacos por sonda enteral
}

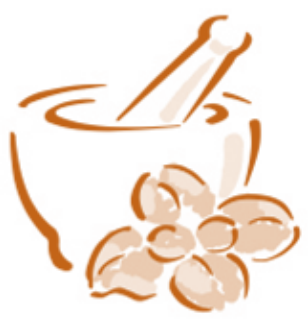

\section{Introducción}

La nutrición enteral por sonda es una técnica de soporte nutricional artificial desarrollada para proveer los requerimientos de energía y de nutrientes a través del tracto digestivo. Está indicada cuando el aparato digestivo es funcionante total o parcialmente y la vía oral no puede utilizarse o resulta insuficiente para cubrir las demandas metabólicas.

La mayor parte de los pacientes que reciben nutrición enteral sufren enfermedades crónicas que precisan tratamiento farmacológico a largo plazo, y en muchos de ellos, este tiene que administrarse mediante la misma sonda al tracto digestivo $\mathrm{Si}$ es bien conocido el hecho de que fármacos y nutrientes pueden interaccionar, no son infrecuentes las dudas que surgen cuando un enfermo necesita un determinado preparado farmacéutico para ser infundido por esta vía. Una administración incorrecta puede originar alteraciones en la eficacia del tratamiento o del soporte nutricional, o comprometer la seguridad del paciente. Complicaciones de otra índole supondrían obstrucción de la sonda por formación de precipitados.

Las sondas pueden ser quirúrgicas y no quirúrgicas. Las sondas quirúrgicas o gastro-enterostomías comunican alguna parte del aparato digestivo (generalmente, estómago o intestino delgado) con el exterior a través de un catéter o sonda que perfora la piel.

Las sondas no quirúrgicas o nasoentéricas consisten en la introducción de una sonda a través de la nariz hasta el estómago o intestino.
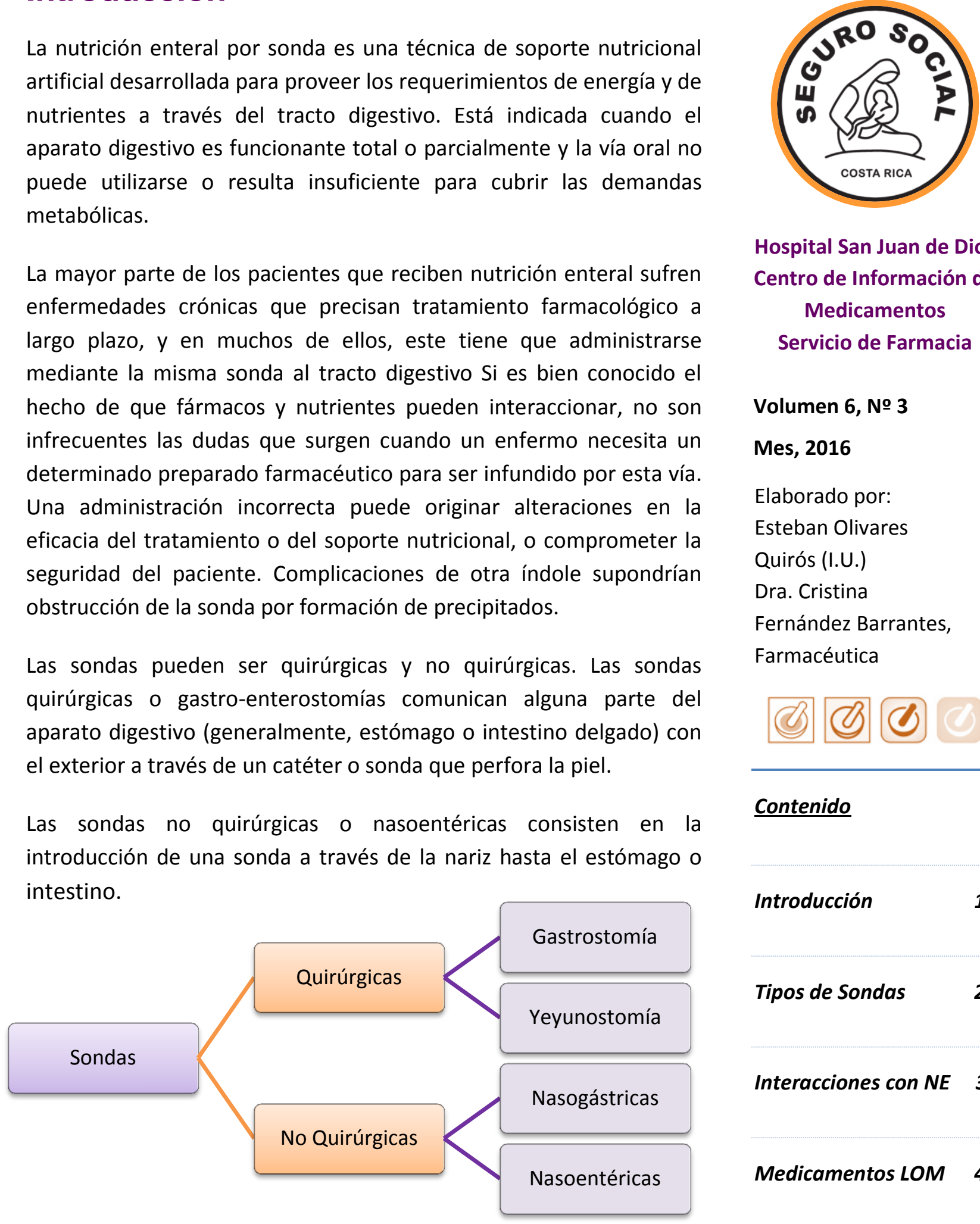

Hospital San Juan de Dios Centro de Información de Medicamentos

Servicio de Farmacia

Volumen 6, № 3

Mes, 2016

Elaborado por:

Esteban Olivares

Quirós (I.U.)

Dra. Cristina

Fernández Barrantes, Farmacéutica

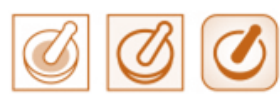

\section{Contenido}

Introducción

Tipos de Sondas

Interacciones con NE 3

Medicamentos LOM 
GI: Gastrointestinal.

NE: Nutrición Enteral.

PEG: Gastrostomía Percutánea

Endoscópica.

SNG: Sonda nasogástrica.

SND: Sonda nasoduodenal

SNY: Sonda nasoyeyunal.

YEG: Yeyunostomía Percutanea

Endoscópica

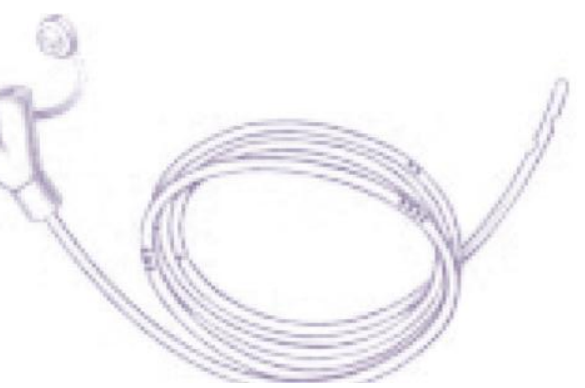

Sonda Nasoentérica

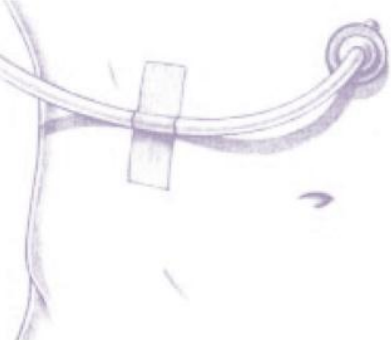

Sonda de Gastrostomía

Sonda nasogástrica (SNG)

Sonda nasoentérica (SND-SNY)

- (más allá del píloro, el extremo distal de la sonda se sitúa en duodeno o en yeyuno)

Gastrostomía (PEG)

•(endoscópica, radiológica o quirúrgica)

Yeyunostomía (YEG)

•(quirúrgica o radiológica)

Sonda de yeyunostomía transgástrica

-(la sonda llega al yeyuno desde una gastrostomía)
El uso de las sondas para administrar medicamentos requiere tener en cuenta distintos factores que pueden modificar la eficacia y seguridad del fármaco, como son: situación de la sonda, forma farmacéutica empleada o interacciones con la nutrición enteral. Para cada medicamento debemos elegir la forma farmacéutica adecuada y realizar una preparación y administración correcta para no obstruir la sonda, conseguir su efectividad, y evitar efectos adversos e interacciones.

Las sondas pueden ser flexibles, rígidas o semirígidas. El extremo proximal queda en el exterior y el distal es el que se introduce en el aparato digestivo. El calibre de la sonda viene indicado en unidades french. El diámetro se calcula multiplicando el número de la sonda por 0.33 (Por ejemplo, una sonda del número 14 tiene un diámetro de $4.6 \mathrm{~mm}$. Los materiales más frecuentemente utilizados son el PVC (para administraciones de 10 - 14 días) y la silicona (administraciones más largas: 2 - 3 meses).

Las sondas que alcanzan la zona postpilórica suelen ser de un calibre más fino que aquellas cuyo extremo distal reposa en estómago, e incluso las sondas de doble luz (con acceso a estómago y a intestino) son mucho más finas en su región distal de lo que es posible observar desde el exterior.

\section{Tipos de sondas}

\section{* Sondas nasoentéricas:}

Son las utilizadas en el sondaje no quirúrgico o nasoentérico. Estas sondas pueden ser: Nasogástricas (el extremo distal está colocado en el estómago), Nasoduodenal (el extremo distal está en el duodeno), Nasoyeyunal (el extremo se encuentra en el yeyuno.)

Las sondas nasogástricas suelen medir entre 76 y $125 \mathrm{~cm}$ de longitud. Las nasoduodenales y yeyunales miden entre 91 y $300 \mathrm{~cm}$. y tienen en su extremo un lastre (generalmente de tungsteno) que ayuda a que la sonda alcance el intestino impulsada por los movimientos peristálticos. 


\section{Sondas de gastro-enterostomía o quirúrgicas:}

Las sondas quirúrgicas deben ser colocadas por el médico mediante acto quirúrgico o endoscópico. Son sondas especiales que suelen tener en su extremo distal un pequeño balón relleno que impide que ésta se salga del estómago o intestino. El extremo externo de la sonda presenta dos entradas: un orificio más amplio, que es a través del cual se administra la medicación y un orificio más pequeño, que es la válvula de seguridad que controla el balón interno y que será revisado periódicamente.

Con respecto a la digestión y a la absorción de nutrientes, cada uno de estos accesos condiciona peculiaridades que han de ser tenidas en cuenta en el momento de prescribir una pauta de nutrición enteral (velocidad de infusión, osmolaridad de la fórmula). Lo mismo ocurre cuando se trata de especificar los cuidados de la sonda (frecuencia de lavado, valoración de la posición).

\section{Interacciones con la Nutrición Enteral}

La NE es una fuente de interacciones con los fármacos, algunos pueden ver alterada su absorción o degradarse en presencia de la NE, mientras que otros verán potenciada su absorción o disminuidos sus efectos adversos sobre la mucosa gástrica.

Cuando haya dudas sobre la compatibilidad, será preferible separar la administración del medicamento de la NE. En el caso de una NE continua se recomienda administrar la medicación quince minutos después de haberla detenido. Para NE discontinuas, la recomendación general es administrar la medicación una hora antes o dos horas después.

Las interacciones pueden ser de varios tipos:

Interacciones
físico-químicas
-Seproduce un
cambio físico,
generalmente
precipitando el
principio
activo, lo que
impide su
absorción y
puede llegar a
cerrar la luz de
la sonda.
También
puede inactivar
nutrientes en
la NE.

\begin{tabular}{|c|}
\hline $\begin{array}{l}\text { Incompatibilida } \\
\text { des de } \mathrm{pH}\end{array}$ \\
\hline $\begin{array}{l}\text { - Los principios } \\
\text { activos con } \mathrm{pH} \\
<3,5 \text { o >10 } \\
\text { pueden } \\
\text { precipitar con } \\
\text { la NE. En } \\
\text { sondas } \\
\text { transpilóricas y } \\
\text { YEG está } \\
\text { contraindicado } \\
\text { todo fármaco } \\
\text { con pH ácido, } \\
\text { ya que podrían } \\
\text { originar } \\
\text { intolerancia. }\end{array}$ \\
\hline
\end{tabular}

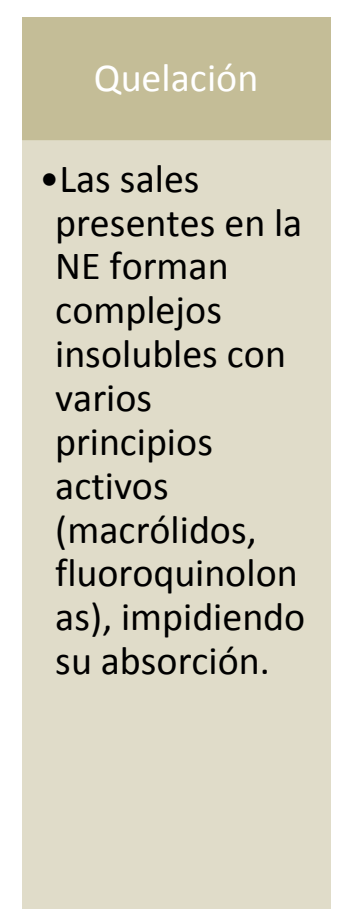

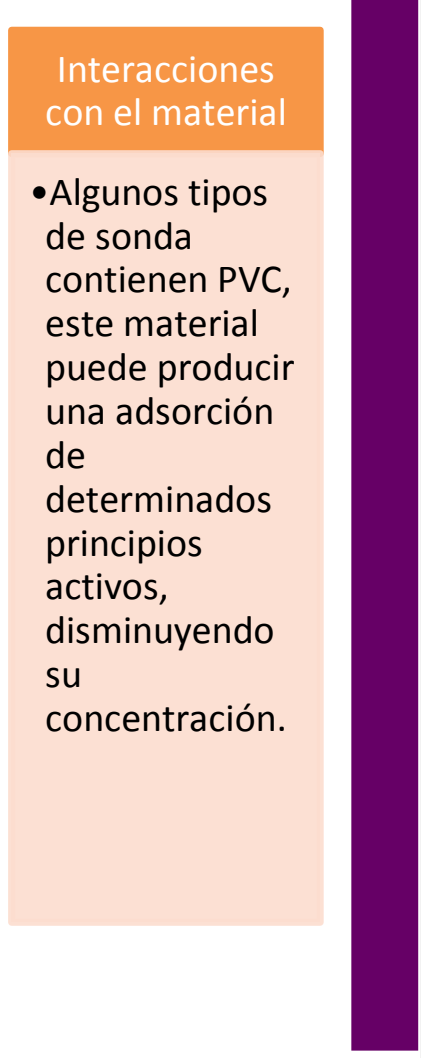




\section{Administración por esta vía de algunos medicamentos LOM}

\begin{tabular}{|c|c|}
\hline ABACAVIR & Comprimidos \\
\hline $\begin{array}{l}\text { Administración por SNG, } \\
\text { PEG, Gastrostomía: }\end{array}$ & SI \\
\hline Compatibilidad NE: & Compatible con la NE \\
\hline Observaciones: & $\mathrm{N} / \mathrm{A}$ \\
\hline
\end{tabular}

\begin{tabular}{ll} 
ACICLOVIR & Comprimidos \\
\hline $\begin{array}{l}\text { Administración por SNG, } \\
\text { PEG, Gastrostomia: }\end{array}$ & SI \\
\hline Compatibilidad NE: & Compatible con la NE \\
\hline Observaciones: & N/A \\
\hline
\end{tabular}

\begin{tabular}{|l|l|}
\hline ALENDRONATO & Comprim. Recubiertos \\
\hline $\begin{array}{ll}\text { Administracion por SNG, } \\
\text { PEG, Gastrostomia: }\end{array}$ & SI \\
\hline Compatibilidad NE: & No Compatible con la \\
\hline Observaciones: & NE. \\
\hline
\end{tabular}

AMIODARONA
Administracion por SNG,
PEG, Gastrostomia:
Compatibilidad NE:
Observaciones:

\begin{tabular}{l} 
AMLODIPINO \\
Administracion por SNG, \\
PEG, Gastrostomia: \\
Compatibilidad NE: \\
\hline Observaciones: \\
\hline
\end{tabular}

\begin{tabular}{l} 
ANASTROZOL \\
Administracion por SNG, \\
PEG, Gastrostomia: \\
\hline Compatibilidad NE: \\
\hline Observaciones:
\end{tabular}

CALCIO ACETATO
Administracion por SNG,
PEG, Gastrostomia:
Compatibilidad NE:
Observaciones:

\begin{tabular}{l} 
CEFALEXINA \\
Administracion por SNG, \\
PEG, Gastrostomia: \\
Compatibilidad NE: \\
\hline Observaciones:
\end{tabular}

\section{Cápsulas Duras}

\section{SI}

No Compatible con la NE

$N / A$

ABACAVIR
Administración por SNG,
PEG, Gastrostomia:
Compatibilidad NE:
Observaciones:

\section{Solución}

NO

N/A

Contiene un elevado \% de sorbitol (33\%).

\section{ASPIRINA \\ Comprimidos \\ Administración por SNG, PEG, Gastrostomia: \\ Compatibilidad NE: \\ Observaciones: \\ SI \\ Compatible con la NE N/A}

\section{ALUMINIO HIDRÓXIDO Suspensión \\ Administracion por SNG, PEG, Gastrostomia: \\ SI \\ Compatibilidad NE: \\ Compatible con la NE \\ Observaciones: \\ N/A}

\begin{tabular}{|c|c|}
\hline AMITRIPTILINA & Comprim. Recubiertos \\
\hline $\begin{array}{l}\text { Administracion por SNG, } \\
\text { PEG, Gastrostomia: }\end{array}$ & SI \\
\hline Compatibilidad NE: & Compatible con la NE \\
\hline Observaciones: & $\mathrm{N} / \mathrm{A}$ \\
\hline
\end{tabular}

AMOXICILINA
Administracion por SNG,
PEG, Gastrostomia:
Compatibilidad NE:
Observaciones:

\section{Comprimidos}

SI

Compatible con la NE

Detener NE para $\uparrow \mathrm{F}$

\section{ATENOLOL \\ Administracion por SNG, PEG, Gastrostomia: \\ Compatibilidad NE: \\ Observaciones:}

Comprimidos

\section{CARBAMAZEPINA \\ Administracion por SNG, PEG, Gastrostomia: \\ Compatibilidad NE: \\ Observaciones:}
SI
Compatible con la NE NE reduce biodisponib.

\section{Comprimidos}

SI

Compatible con la NE No sondas de PVC

\begin{tabular}{l} 
CIPROFLOXACINO \\
Administracion por SNG, \\
PEG, Gastrostomia: \\
Compatibilidad NE: \\
\hline Observaciones:
\end{tabular}

\section{Comprimidos}

SI

No Compatible con la NE

Suspensión es elección 


\begin{tabular}{ll}
\hline CLARITROMICINA & Comprimidos Lib. Prol. \\
\hline $\begin{array}{l}\text { Administración por SNG, } \\
\text { PEG, Gastrostomia: }\end{array}$ & NO \\
\hline $\begin{array}{l}\text { Compatibilidad NE: } \\
\text { Observaciones: }\end{array}$ & N/A \\
\hline
\end{tabular}

\begin{tabular}{ll}
\hline ENALAPRIL & Comprimidos \\
\hline $\begin{array}{l}\text { Administracion por SNG, } \\
\text { PEG, Gastrostomia: }\end{array}$ & SI \\
\hline Compatibilidad NE: & Compatible con la NE \\
\hline Observaciones: & N/A \\
\hline
\end{tabular}

\begin{tabular}{l} 
FENITOÍNA \\
Administracion por SNG, \\
PEG, Gastrostomia: \\
Compatibilidad NE: \\
\hline Observaciones:
\end{tabular}

\begin{tabular}{l} 
FLUCONAZOL \\
Administracion por SNG, \\
PEG, Gastrostomia: \\
Compatibilidad NE: \\
\hline Observaciones:
\end{tabular}

\begin{tabular}{l} 
FUROSEMIDA \\
Administracion por SNG, \\
PEG, Gastrostomia: \\
Compatibilidad NE: \\
\hline Observaciones:
\end{tabular}

GLIBENCLAMIDA
Administracion por SNG,
PEG, Gastrostomia:
Compatibilidad NE:
Observaciones:

\begin{tabular}{l} 
IBUPROFENO \\
Administracion por SNG, \\
PEG, Gastrostomia: \\
\hline Compatibilidad NE: \\
Observaciones: \\
\hline
\end{tabular}

ISOSORBIDE
Administracion por SNG,
PEG, Gastrostomia:
Compatibilidad NE:
Observaciones:

\section{Comprimidos/Cápsulas}

SI

Compatible con la NE

Puede obstruir sonda

\section{Cápsulas duras/Suspens}

SI

Compatible con la NE

Puede obstruir sonda

\section{Comprimidos}

SI

Compatible con la NE

N/A

\section{Comprimidos}

SI

No Compatible con NE N/A

\section{Comprimidos}

SI

Compatible con la NE Administrar con NE

\section{Comprimidos}

SI

No Compatible con NE Usar 1 hora antes de NE

\begin{tabular}{ll} 
EFAVIRENZ & Comprimidos/Cápsulas \\
\hline $\begin{array}{l}\text { Administración por SNG, } \\
\text { PEG, Gastrostomia: }\end{array}$ & SI \\
\hline Compatibilidad NE: & $\begin{array}{l}\text { No Compatible con la } \\
\text { NE }\end{array}$ \\
\hline Observaciones: & N/A
\end{tabular}

\section{ESPIRONOLACTONA Comprimidos \\ Administracion por SNG, PEG, Gastrostomia: \\ Compatibilidad NE: \\ Observaciones: \\ SI \\ Compatible con la NE N/A}

\section{FEXOFENADINA}

Comprimidos Recub.

Administracion por SNG,

PEG, Gastrostomia:

Compatibilidad NE:

Observaciones:

\section{SI}

No Compatible con la NE

No se recomienda

\begin{tabular}{l} 
FLUOXETINA \\
Administracion por SNG, \\
PEG, Gastrostomia: \\
Compatibilidad NE: \\
\hline Observaciones:
\end{tabular}

Comprimidos/Cápsulas

SI

Compatible con la NE Solución es elección

\begin{tabular}{l} 
GEMFIBROZILO \\
Administracion por SNG, \\
PEG, Gastrostomia: \\
\hline Compatibilidad NE: \\
\hline Observaciones:
\end{tabular}

\section{Comprimidos}

SI

No Compatible con la NE

Usar 1 hora antes de NE

\begin{tabular}{l} 
HIDROCLOROTIAZIDA \\
Administracion por SNG, \\
PEG, Gastrostomia: \\
\hline Compatibilidad NE: \\
\hline Observaciones:
\end{tabular}

\begin{tabular}{l} 
IRBESARTÁN \\
Administracion por SNG, \\
PEG, Gastrostomia: \\
\hline Compatibilidad NE: \\
Observaciones:
\end{tabular}

\section{IVERMECTINA}

Administracion por SNG,

PEG, Gastrostomia:

Compatibilidad NE:

Observaciones:

\section{Comprimidos}

SI

Compatible con la NE $10 \mathrm{~min} \mathrm{p} /$ disolverse

\section{Comprimidos Recub.}

SI

Compatible con la NE N/A

\section{Comprimidos}

SI

N/A

N/A 


\begin{tabular}{l} 
LAMIVUDINA \\
Administración por SNG, \\
PEG, Gastrostomía: \\
Compatibilidad NE: \\
\hline Observaciones:
\end{tabular}

LEVOTIROXINA
Administracion por SNG,
PEG, Gastrostomia:
Compatibilidad NE:
Observaciones:

\section{LOVASTATINA}

Administracion por SNG,
PEG, Gastrostomia:
Compatibilidad NE:
Observaciones:

METILDOPA
Administracion por SNG,
PEG, Gastrostomia:
Compatibilidad NE:
Observaciones:

NITROFURANTOÍNA
Administracion por SNG,
PEG, Gastrostomia:
Compatibilidad NE:
Observaciones:

\section{RISPERIDONA}

Administracion por SNG,

PEG, Gastrostomia:

Compatibilidad NE:

Observaciones:

\section{MICOFENOLATO MOFET. \\ Administracion por SNG, \\ PEG, Gastrostomia: \\ Compatibilidad NE: \\ Observaciones:}

\section{Comprimidos}

SI

Compatible con la NE N/A

\section{Comprimidos}

\section{SI}

No Compatible con NE Incompatible con NE fibra. Fotosensible

\section{Comprimidos}

\section{SI}

Compatible con la NE No con NE con fibra

\section{Comprimidos}

\section{SI}

No Compatible con NE Usar 1 hora antes

\section{Comprimidos}

\section{SI}

No Compatible conNE Elección en SNY, YEG

\section{Comprimidos}

\section{SI}

Compatible con NE N/A

\section{Comprimidos}

\section{NO}

No Compatible con NE Cambiar a suspensión

\begin{tabular}{l} 
TRIMETOPRIM \\
SULFAMETOXAZOL \\
Administracion por SNG, \\
PEG, Gastrostomia: \\
Compatibilidad NE: \\
\hline Observaciones:
\end{tabular}

\section{Comprimidos}

\section{SI}

No Compatible con NE Usar 1 hora antes de NE

\section{LAMOTRIGINA \\ Administración por SNG, \\ PEG, Gastrostomia: \\ Compatibilidad NE: \\ Observaciones: \\ Comprimidos dispersab \\ SI \\ Compatible con la NE N/A}

\section{LOPINAVIR/RITONAVIR Comprimidos recub. \\ Administracion por SNG, PEG, Gastrostomia: \\ Compatibilidad NE: No Compatible con NE \\ Observaciones: \\ Cambiar a Solución}

\section{METFORMINA \\ Administracion por SNG, \\ PEG, Gastrostomia: \\ Compatibilidad NE: \\ Observaciones:}

METOTREXATO
Administracion por SNG,
PEG, Gastrostomia:
Compatibilidad NE:
Observaciones:

RIFAMPICINA
Administracion por SNG,
PEG, Gastrostomia:
Compatibilidad NE:
Observaciones:

\section{TACRÓLIMUS}

Administracion por SNG,

PEG, Gastrostomia:

Compatibilidad NE:

Observaciones:

\section{Comprimidos}

SI

No Compatible con NE Manipulación especial

\section{Comprimidos Recub.}

\section{SI}

No Compatible con NE Fotosensible

\section{Cápsulas Duras}

\section{SI}

No Compatible con NE No usar sondas PVC

\section{TENOFOVIR \\ Administracion por SNG, PEG, Gastrostomia: \\ Compatibilidad NE: \\ Observaciones:}

\section{Comprimidos}

\section{SI}

Compatible con la NE Administrar con NE

\section{ZIDOVUDINA}

Cápsulas duras

Administracion por SNG,

PEG, Gastrostomia:

Compatibilidad NE:

Observaciones:

\section{SI}

No Compatible con NE Usar 1 hora antes de NE 


\section{Alertas de Farmacovigilancia}

Analgésicos Opioides: La FDA, está advirtiendo acerca de varios problemas de seguridad con toda la clase de los analgésicos opiáceos. Estos riesgos de seguridad incluyen interacciones potencialmente nocivas con muchos otros medicamentos, problemas con las glándulas suprarrenales y disminución de los niveles de hormonas sexuales. Se están exigiendo cambios a la etiqueta de todos los medicamentos opiáceos para advertir sobre estos riesgos. http://www.fda.gov/Drugs/DrugSafety/ucm492745.htm, consultado el 22-03-2016

Corticosteroides inhalados: la Agencia Europea del Medicamento (EMA), estudió el riesgo de neumonía en pacientes en tratamiento con corticosteroides inhalados para el tratamiento del EPOC. Esta evaluación concluyó que sí existe un riesgo aumentado pero que aún así son mayores los beneficios de continuar con el tratamiento. Se recomienda que se haga una actualización del etiquetado de los productos e insistir que hasta el momento no es necesario realizar alguna medida regulatoria adicional.

http://www.ema.europa.eu/ema/index.jsp?curl=pages/news and events/news/2016/03/news detail 002 491.jsp\&mid=WC0b01ac058004d5c1 consultado el 01-04-2016

Aflibercept: La EMA emite alerta sobre el riesgo de desarrollar osteonecrosis mandibular en pacientes en tratamiento con aflibercept. Este medicamento está indicado, en combinación con irinotecan/5fluorouracilo/ácido folínico, en adultos con cáncer colorrectal metastásico resistente a o que haya progresado, después de un régimen con oxaliplatino. La recomendación regulatoria incluye que antes de iniciar tratamiento es conveniente que el paciente se realice un examen dental y mantenga un cuidado dental preventivo apropiado. En la medida de lo posible deberán evitarse los procedimientos dentales invasivos especialmente si hayan recibido o estén recibiendo bifosfonatos intravenosos. http://www.aemps.gob.es/informa/notasInformativas/medicamentosUsoHumano/seguridad/2016/NI-

MUH FV 04-aflibercept-zaltrap.htm consultado el 16-03-2016

\section{Referencias consultadas}

Arenaza P, A; Arias F, L; Benítez M, M; et al. Guía de Administración de Medicamentos por Sondas de Alimentación Enteral. Imprenta Hospital Clínico San Carlos. Madrid: 2012. Pp 1-280 (ISBN: 978-84-695-5114-1)

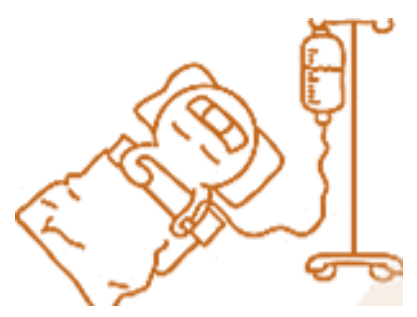

* Izco N, Creus N, Massó J, Codina C, Ribas J. Incompatibilidades fármaconutrición enteral: recomendaciones generales para su prevención. Farm Hosp. 2001 (25)1:13-24.

* Sánchez G, E; Rivas R, R; Romero A, M; et al. Guía para la administración segura de medicamentos vía oral. Artes Gráficas Bonanza. Hospital Juan Ramón Jiménez, Huelva. España: 2009. (ISBN: 97884-692-7044-8)

* Otero MJ, Martín R, Robles MD, Codina C. Errores de medicación. En: Bonal Fagas J, DomínguezGil Hurle A, Gamundi Planas MC, Napal Lecumberri V, Valverde Molina E. Farmacia Hospitalaria. Fundación Española de Farmacia Hospitalaria. 10 Edición. Madrid. 2004:713-747.

Ventura López $\mathrm{M}$ et al. Nutrición enteral: guía de dietas y fármacos para administración por sonda. Servicio de Farmacia Hospital General Universitario Morales Meseguer. Murcia Segunda Edición 2002. 